\title{
Linforragia cervical derecha: A propósito de un caso
}

\author{
M.A. Sicilia Gutiérrez¹, LM. Capitán Cañadas¹, S. Martínez-Villalobos Castillo², \\ J. Fernández Solís², D. Sánchez López ${ }^{2}$, E. Valencia Laseca ${ }^{3}$
}

\begin{abstract}
Resumen: La linforragia cervical en el lado derecho es una complicación bastante infrecuente en los procedimientos quirúrgicos del cuello, con una incidencia, según las series, establecida en torno a un 0,50$0,75 \%$. Pueden ser producidos y drenados a través de la fístula de 2 a 4 litros de linfa al día, hecho que puede plantear dificultades en el mantenimiento del balance de fluidos corporales e importantes desórdenes metabólicos y electrolíticos. Se presenta un caso en un paciente de 57 años de edad, diagnosticado de carcinoma epidermoide de lengua, catalogado como $\mathrm{CT}_{3} \mathrm{~N}_{1} \mathrm{M}_{0}$, sometido a tumorectomía más vaciamiento cervical funcional (VCF) derecho y que presentó dicha complicación a los 6 días postcirugía.
\end{abstract}

Palabras clave: Linforragia Cervical; Disección Cervical Funcional; Complicación.

\begin{abstract}
Chyle fistula in the right side is a quite infrequent complication in the surgical procedures of the neck, with an incidence stablished conceming 0,50-0,75\%, according to the series. They can be produced and drained through the fistula around 2-4 liters of lymph per day, fact that can create difficulties in the maintenance of the corporal fluids balance and important metabolic and electrolytic disorders. We present a case that appears in a 57 years old man diagnosed of epidermoid carcinoma of the tongue, catalogued like $\mathrm{cT}_{3} \mathrm{~N}_{1} \mathrm{M}_{0}$, submitted for tumour al resection and functional neck dissection with the above mentioned complication presented to the 6 days post surgery.
\end{abstract}

Key words: Cervical Chyle Fistula; Functional Neck Dissection; Complication.

\section{Introducción}

Se entiende por linforragia cervical la salida anormal de linfa fuera del ductus torácico, secundario habi-

\footnotetext{
1 Médico Residente

2 Médico Adjunto

3 Jefe de Servicio

Servicio de Cirugía Oral y Maxilofacial. Centro de Rehabilitación y Traumatología. Hospital Universitario «ingen de las Nieves». Granada, España
}

\section{Correspondencia:}

Marco Antonio Sicilia Gutiérrez

Servicio de Cirugía Oral y Maxilofacial.

Centro de Rehabilitación y Traumatología.

Hospital Universitario «irgen de las Nieves».

Ctra. de Jaén s/n.

18014 Granada, España.

E-mail: masigut@yahoo.es. tualmente a un traumatismo quirúrgico, hacia las estructuras anatómicas del cuello. Es una complicación relativamente infrecuente pero importante por su trascendencia en los procedimientos quirúrgicos del cuello. Conlet (1979) estableció la incidencia de dicho proceso entre un 1-2\%, apareciendo la mayoría de los casos en las disecciones cervicales del lado izquierdo vs. un $25 \%$ de casos que ocurrían en el lado derecho..$^{1-8}$

Pueden ser producidos y drenados a través de la fístula de dos a cuatro litros de linfa al día. ${ }^{9}$ Este hecho puede plantear problemas en relación al mantenimiento del balance de fluidos corporales, a la nutrición e importantes desordenes metabólicos y electrolíticos, a lo que se le añade una disminución en la capacidad inmunitaria del paciente al producirse una disminución de glóbulos blancos, sobre todo de linfocitos, que puede hacer al 


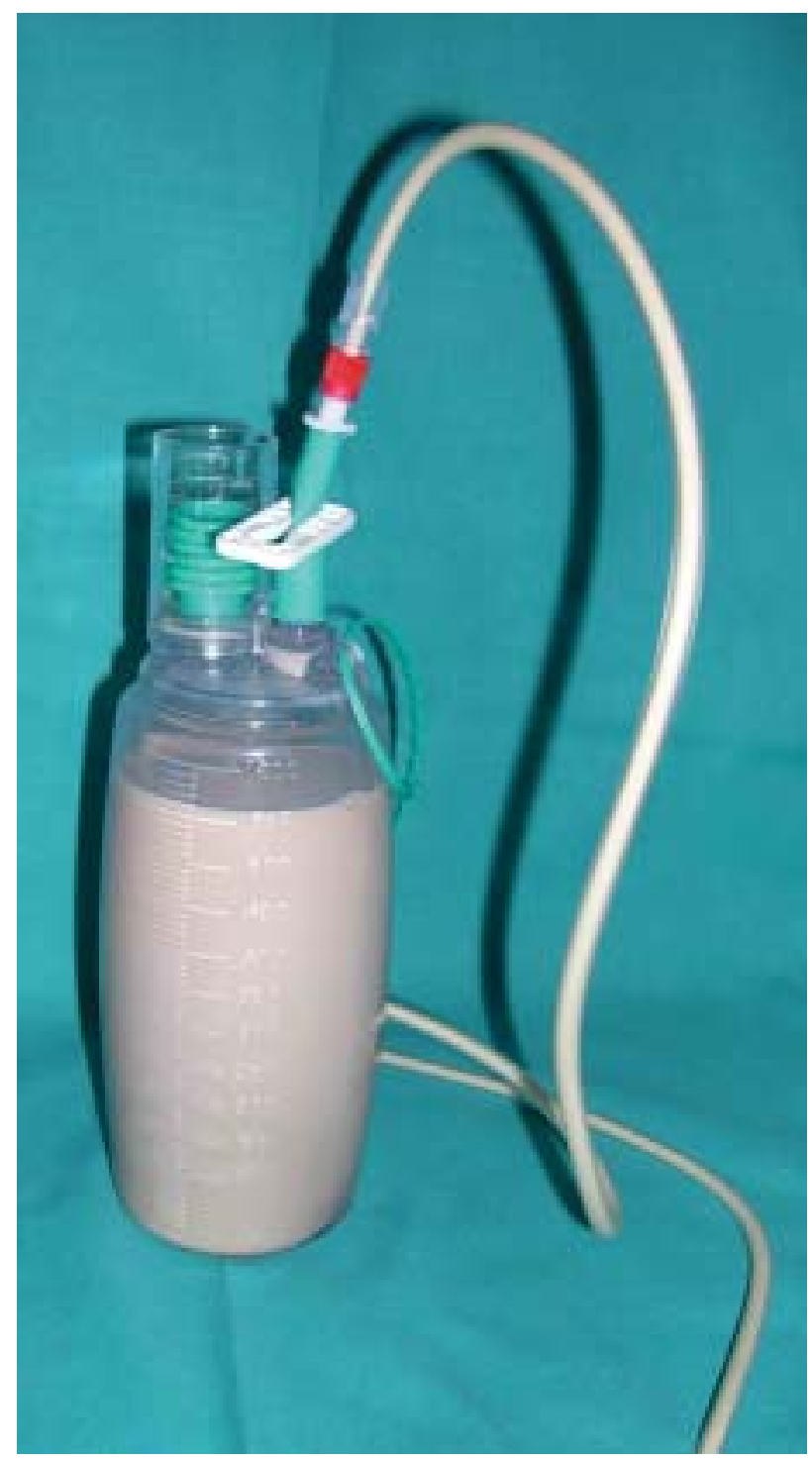

Figura 1. Bote de redón donde se visualiza el acúmulo de líquido lechoso de 24 horas de evolución. Corresponde a linforragia sobreinfectada.

paciente más susceptible a la infección en el postoperatorio inmediato. ${ }^{10}$ Todo ello puede favorecer un fracaso en la cicatrización (Fig. 1).

Presentamos un caso clínico intervenido en nuestro Servicio, además de revisar y actualizar las diferentes aproximaciones terapéuticas de esta complicación.

\section{Caso clínico}

Varón de 57 años de edad con antecedentes personales de hernia de hiato y vagotomía más piloroplastia por ulcus gástrico que consulta por tumoración úlcero-infiltrante de tercio posterior de lengua y pilar anterior amigdalino derechos, con biopsia diagnóstica de carcinoma epidermoide moderada-

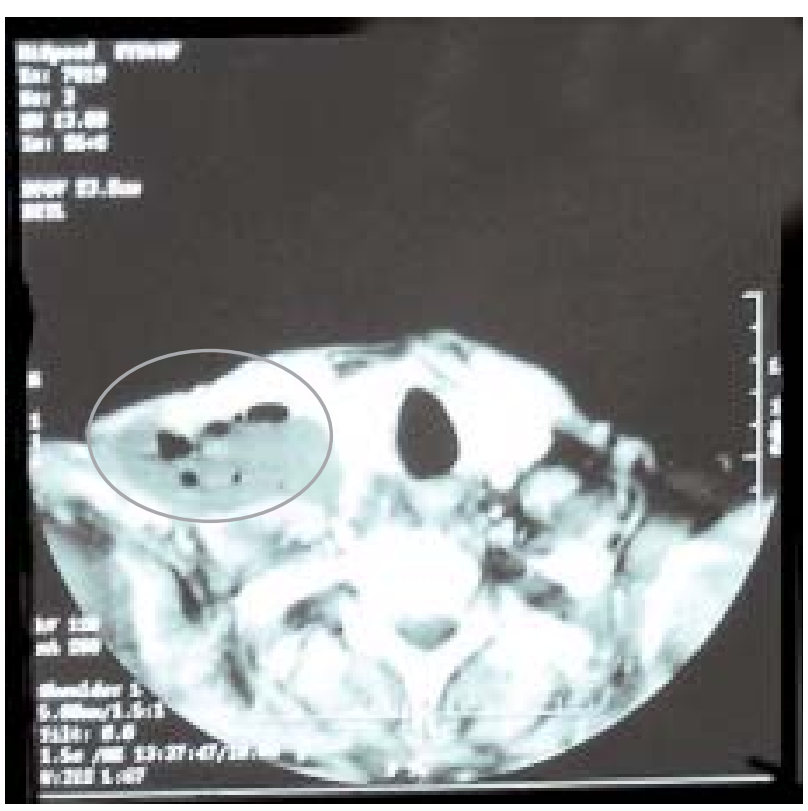

Figura 2. Imagen TAC donde se observa la colección a nivel supraclavicular derecho.

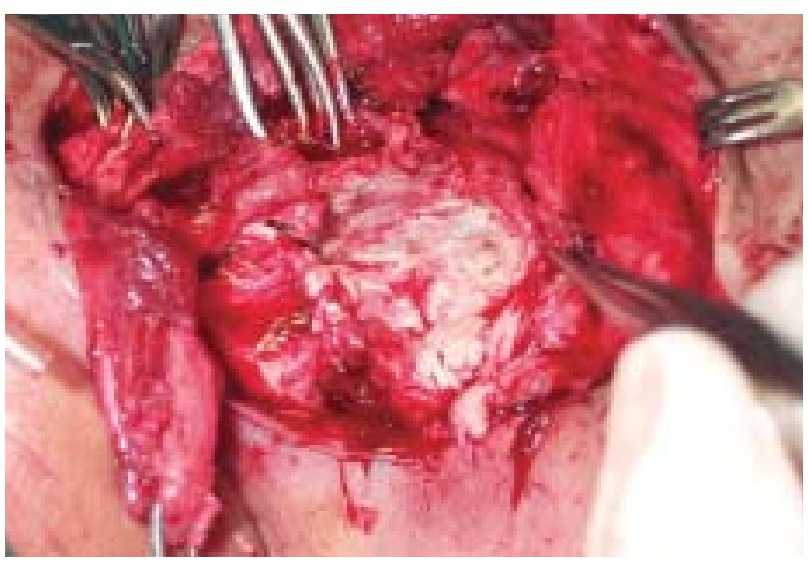

Figura 3. Imagen intraoperatoria, donde se observa la aparición de secreción lechosa en el campo quirúrgico.

mente diferenciado. Catalogado tras estudio TC como $\mathrm{CT}_{3} \mathrm{~N}_{1} \mathrm{M}_{\mathrm{x}}$, se trata quirúrgicamente realizándose tumorectomía con márgenes y VCF derecho.

El postoperatorio cursa tórpidamente, con dehiscencia de sutura intraoral pero sin evidencia de comunicación orocervical, por lo que se deja granular por segunda intención permaneciendo el paciente con nutrición enteral mediante sonda nasogástrica (SNG). Al sexto día del postoperatorio se detecta discreta colección supraclavicular que es drenada tras retirada de algunos agrafes, obteniéndose una secreción blanquecina-lechosa compatible con secreción linfática sobreinfectada. Se insertó drenaje aspirativo, se instauró antibioterapia de amplio espectro vía parenteral y el paciente continuó con medidas dietéticas (nutrición enteral por SNG) y posturales (decúbito a $45^{\circ}$ ) así como con vendaje com- 


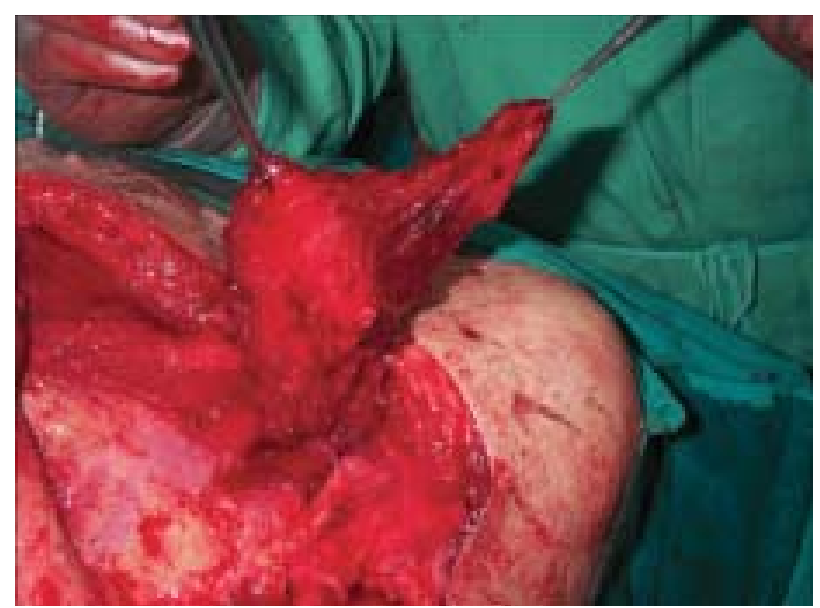

Figura 4. Imagen intraoperatoria, obteniéndose el colgajo miofascial de rotación de músculo pectoral mayor para sellado del defecto.

presivo enérgico sobre región supraclavicular, evidenciándose una progresiva disminución del débito de la secreción cervical. El estudio bioquímico confirmó la sospecha de colección linfática. Doce días después de ser detectada, la linforrea quedó resuelta y el paciente fue dado de alta hospitalaria sin más complicaciones.

Sin embargo a los 12 días del alta, 30 desde la intervención, el paciente reingresa en el hospital por presentar una nueva colección supraclavicular derecha (Fig. 2). Se reinterviene quirúrgicamente practicándose drenaje, limpieza de cavidad quística, coagulación de vasos linfáticos y colgajo por deslizamiento de músculo pectoral mayor para cobertura del lecho quirúrgico (Figs. 3 y 4). El estudio bioquímico confirmó la recidiva de la linforragia. En el postoperatorio se aplicaron las medidas dietéticas, posturales y cura compresiva antes citadas. El paciente evolucionó de forma favorable siendo dado de alta, previo control TC, a los diez días de la reintervención. Pasó a recibir RT complementaria sin nuevas incidencias.

\section{Discusión}

La incidencia de la linforragia como complicación en la cirugía del cuello varía entre un 1-2,5\%,1-3 y habitualmente se produce tras las disecciones cervicales del lado izquierdo, por ser esa la zona donde con más frecuencia drena el conducto torácico su contenido a la vena yugular intema. ${ }^{10} \mathrm{El}$ ductus habitualmente desemboca a $1 \mathrm{~cm}$. de la unión entre vena yugular interna izquierda y la vena subclavia, aunque esta anatomía presenta considerables variaciones, como muestran los estudios de Langford y cols. ${ }^{4}$ y Shimada y cols. ${ }^{5}$ (Fig. 5).

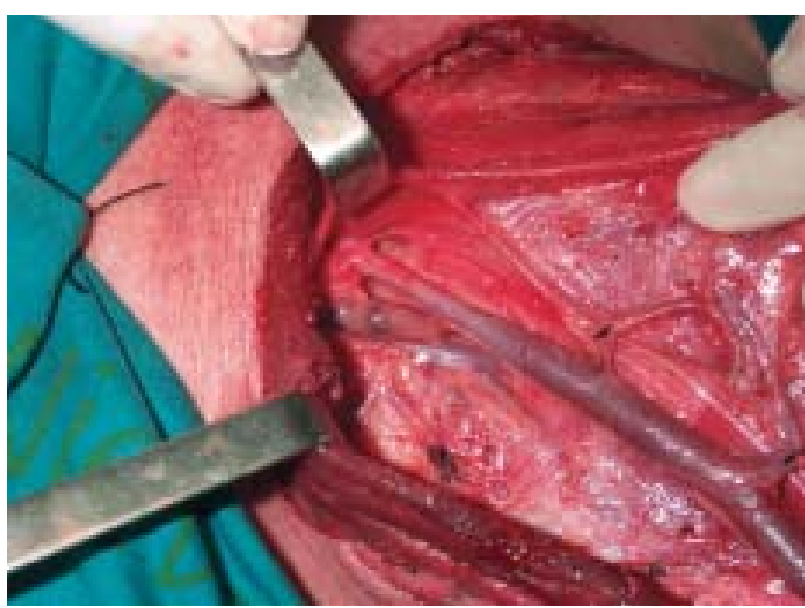

Figura 5. Detalle anatómico de la inserción del conducto torácico en la vena yugular interna izquierda.

El diagnóstico se realiza bien en el acto operatorio o bien postoperatoriamente. En el primer caso la linfa se reconoce macroscópicamente como una sustancia lechosa-oleosa en el campo operatorio. Otras veces, el primer signo que sugiere la presencia de fístula, es un tacto grasiento en los guantes del cirujano. ${ }^{6}$

$\mathrm{Si}$ se sospecha la presencia de linforragia, puede ser confirmada con la ayuda del anestesiólogo, aplicando una presión positiva continua en las vías aéreas y dejando al enfermo en posición de Trendelenburg. ${ }^{7}$ Postoperatoriamente, la linfa aparecerá en el bote de drenaje, aunque si su volumen es mínimo, puede no ser detectado inicialmente al mezclarse con la sangre, imponiéndose entonces realizar un análisis bioquímico de la muestra para confirmar el diagnóstico.

La linfa es un líquido transparente 0 amarillento de reacción alcalina, opalescente por la presencia de partículas grasas. Microscópicamente consta de una porción líquida, con presencia de quilomicrones, y otra celular, fundamentalmente linfocitos. En presencia de sobreinfección, las características macroscópicas y microscópicas pueden variar significativamente (Fig. 1).

En cuanto al manejo terapéutico, está universalmente aceptado que la mejor medida es la prevención. ${ }^{7}$ Habitualmente, debe evitarse el esfuerzo de encontrar la fístula linfática, ya que puede resultar en un mayor destrozo de tejidos. ${ }^{8} \mathrm{Si}$ la fístula se identifica intraoperatoriamente, debe tratarse rápidamente, mediante la ligadura del conducto con sutura de 3 ó 4-0 no reabsorbible. ${ }^{6}$

El siguiente paso terapéutico es iniciar medidas posturales y médicas, incluyendo entre las primeras, el posicionamiento del paciente en decúbito a $45^{\circ} \mathrm{y}$ la puesta de un drenaje aspirativo y un vendaje compresivo en la zona quirúrgica. ${ }^{3}$ 
Como medidas dietéticas, Martin y cols. ${ }^{9}$ proponen una dieta que incluya una mezcla de triglicéridos de cadena media (los cuales se absorben sin necesidad de síntesis de quilomicrones y pasan directamente al sistema porta), minerales, ácido fólico y suplementos vitamínicos, obteniendo un resultado satisfactorio en la resolución de la fístula.

Kassel y cols. ${ }^{8}$ desarrolla la escleroterapia con agentes como el talco o la tetraciclina a través del catéter de drenaje o bien percutáneamente como medida alternativa a la hospitalización prolongada o la reexploración quirúrgica.

Casler y Brietzke, ${ }^{11}$ en 1998 describieron un caso en el cual existía una gran pérdida de contenido linfático (mas de $2000 \mathrm{ml}$. de fluido en $24 \mathrm{~h}$ ) a través de una fístula tras una disección cervical radical izquierda, y que no respondió a las medidas conservadoras iniciales, necesitando una reintervención quirúrgica y la resolución del problema mediante un injerto libre de grasa submental sobre la zona de salida del ductus, ofreciendo esta medida como una alternativa en las fístulas cervicales que producen gran débito.

Spiro y cols., ${ }^{3}$ en una revisión de 16 pacientes publicada en 1990, aboga por un preciso conocimiento de la anatomía linfática cervical y sus variaciones como el mejor procedimiento para evitar la linforragia cervical, aconsejando una reintervención quirúrgica en aquellos casos en los que el drenaje exceda de los 600 cc por día.

Otros métodos utilizados en la reparación quirúrgica de la fístula linfática y descritos en la literatura, son el uso de pegamento de fibrina, ${ }^{2,12}$ y el sellado del defecto mediante colgajos musculares.2,12,13

La somatostatina, en perfusión contínua a dosis de $6 \mathrm{mgr} /$ día, se ha mostrado como una terapéutica eficaz y resolutiva en algunos casos de fístulas de alto débito. ${ }^{13,14} \mathrm{Su}$ mecanismo de acción es complejo y no bien conocido, recomendándose la monitorización estrecha de las glucemias durante el tratamiento y una retirada en «escalera»al finalizar el mismo. ${ }^{15}$

Para linforragias mantenidas que persistan más de 30 días, o para aquellos casos donde la cantidad de débito a través del drenaje sea importante, mayor de $1000 \mathrm{ml}$. en $24 \mathrm{~h}$, la medida definitiva es intentar la ligadura del conducto torácico en la cavidad torácica, siendo también esta actitud la elegida cuando la linforragia va asociada a otras complicaciones, como la formación de quilotórax.7,10

\section{Conclusiones}

El tratamiento de la linforragia cervical en general, y en especial en el lado derecho, como com- plicación de las disecciones cervicales es hoy día un tema a debatir. Estamos de acuerdo que la mejor medida es la prevención, incluyendo un exhaustivo conocimiento por parte del cirujano de la anatomía linfática cervical y de sus variaciones.

Es de resaltar que la visualización de esta complicación en el campo quirúrgico es difícil, descubriéndose la mayoría de las veces de forma inesperada en el postoperatorio inmediato.

$\mathrm{Si}$ se detecta en el acto quirúrgico, intentaremos su resolución intraoperatoriamente, evitando grandes manipulaciones en la zona que puedan provocar mayores desgarros. En la mayoría de los casos, esta medida es difícil de realizar y no se consigue con éxito, por lo que el siguiente paso es iniciar medidas conservadoras en el postoperatorio inmediato, incluyendo vendaje compresivo cervical, reposo en cama a $45^{\circ}$ y nutrición enteral, con dieta libre de grasas y enriquecida en triglicéridos de cadena media, proteínas y suplementos vitamínicos.

La terapéutica con somatostatina, si bien no rigurosamente contrastada, pudiera ser una alternativa previa a la reintervención en pacientes de alto riesgo quirúrgico. Una estrecha monitorización endocrino-metabólica se hace imprescindible en estos casos.

Para linforragias mantenidas en el tiempo, de más de 30 días y que no responden al tratamiento conservador, para aquellas donde existe un gran débito (mayor de $1000 \mathrm{ml}$ por día) y para aquellas que asocien otras complicaciones, como el quilotórax, defendemos la pronta reintervención quirúrgica del paciente y la ligadura del ductus torácico.

\section{Bibliografía}

1. Strong EW, Henschke UK, Nickson JJ, y cols. Preoperative $\mathrm{X}$-ray therapy as an adjunct to radical neck dissection. Cancer 1966;16:1509-16.

2. Fitz-Hugh GS, Cowgill R. Chylous fistula. Complication of neck dissection. Arch Otolaryngol 1970;91:5437.

3. Spiro JD, Spiro RH, Strong EW. The management of chyle fistula. Laryngoscope 1990;100:771-4.

4. Langford RJ, Daudua AT, Makins TJ. A morphological study of the thoracic duct at the jugulo-subclavian junction. J Cranio Maxillofac Surg 1999;27:100-4.

5. Shimada K, Sato I. Morphological and histological analysis of the thoracic duct at the jugulo-subclavian junction in Japanese cadavers. Clin Anat 1997;10:163-2.

6. Lapp GC, Brown DH, Gullane PJ, y cols. Thoracoscopic management of chylous fistulae. Am J Otolaryngol 1998; 19:257-62.

7. Hehar SS, Bradley PJ. Management of chyle leaks. Curr Opin Otolaryngol Head Neck Surg 2001;9:120-5. 
8. Kassel RN, Havas TE, Gullane P. The use of topical tetracycline in the mamagement of persistent chylous fistulae. J Otolaryngol 1987;3:174-8.

9. Martin $\mathrm{CH}$, Marinho AE, Browun DM. Medium chain triglycerides in the management of chylous fistulae following neck dissection. Br J Oral Maxillof Surg 1993;31:236-8.

10. Rollón A, Salazar Cl, Mayorga F, y cols. Severe cervical chyle fistula after radical neck dissection. Int J Oral Maxillofac Surg 1996;25:363-5.

11. Casler JD, Brietzke SE. Repair of a high-output chylous fistula with a free fat graft. Laryngoscope 1998;108:938-40.
12. de Gier HW, Balm AJ, Brunung PF, y cols. Systematic approach to the treatment of chylous leakege after neck dissection. Head Neck 1996;18:347-51.

13. Ulibarri JI, Sanz Y, Fuentes C, y cols. Reduction of lymphorrhagia from ruptured thoracic duct by somatostatin. Lancet 1992;39:491-2.

14. Pelizzo MR, Toniato A, Piotto A, y cols. Somatostatin in the treatment of lymphorrhea after lateral cervical dissection. Minerva Chir 1992;47:1485-7.

15. Collard JM, Laterre PF, Boemer F y cols. Conservative treatment of postsurgical lymphatic leaks with somatostatin14. Chest 2000;117:902-5. 